\title{
Reply to the letter: re: Beneath the tip of the iceberg: using electronic referrals to map the unquantified burden of clinical activity in a urology service
}

\author{
Deepak Batura ${ }^{1}$
}

Published online: 23 January 2017

(C) Springer Science+Business Media Dordrecht 2017

\section{Editor,}

We thank Mr Floyd for his interest in our paper and his insightful comments on our work [1, 2]. In their paper, Floyd et al. [3] studied only nonemergency elective referrals. Even accounting for this, it is interesting that the average number of urological consultations sought was higher in our study. The increase in numbers may well be a reflection on the ever growing workload in urology and underscores the importance of capturing referral data accurately.

We note the author's comments on our e-referrals for preoperative issues $(0.3 \%)$. These referrals were from other surgical teams that faced an unresolved urological condition and had sought our inputs. The relatively small percentage of referrals that arose for an elevated PSA (0.8\%) in our study might well be because we have also included emergency e-referrals, which were as many as $46 \%$ of our study population. As we focussed our research only on process analysis, we did not examine outcomes such as urological operations arising from referred patients. Thus, the study by Floyd et al. [3] provides useful information in that context.

We agree with the author that data on the time spent managing these additional patients would be useful to know. Apart from managing demand by provisioning for an adequate workforce, it would also help in ensuring equitable financial reimbursements.

Your's sincerely,

Mr Deepak Batura on behalf of the authors

Compliance with ethical standards

Conflict of interest None.

\section{References}

1. Floyd MS (2016) Reply to the letter: re: Beneath the tip of the iceberg: using electronic referrals to map the unquantified burden of clinical activity in a urology service. Int Urol Nephrol. doi:10.1007/s11255-016-1412-y

2. Batura D, Hashemzehi T, Lee T, Mahbubani K, Ally M, Figaszewska MJ, Kavia R (2016) Beneath the tip of the iceberg: using electronic referrals to map the unquantified burden of clinical activity in a urology service. Int Urol Nephrol 48:1751-1755

3. Floyd MS Jr, Majumdar P, Seipp C, Anandaram PS, De Bolla AR (2010) Urology consultations: the hidden workload? Bull R Coll Surg Engl 92:358-361
Deepak Batura

deepakbatura@gmail.com

1 London North West Healthcare NHS Trust, Watford Road, Harrow, London HA1 3UJ, UK 